UCRL-JC-130160

PREPRINT

\title{
Turbulence Studies in Tokamak Boundary Plasmas with Realistic Divertor Geometry
}

\author{
X.Q. Xu \\ R.H. Cohen \\ G.D. Porter \\ T.D. Rognlien \\ D.D. Ryutov \\ J.R. Myra \\ D.A. D'Ippolito \\ R. Moyer
}

This paper was prepared for submittal to

17th IAEA Fusion Energy Conference

Yokohama, Japan

October 19-24, 1998

October 14, 1998

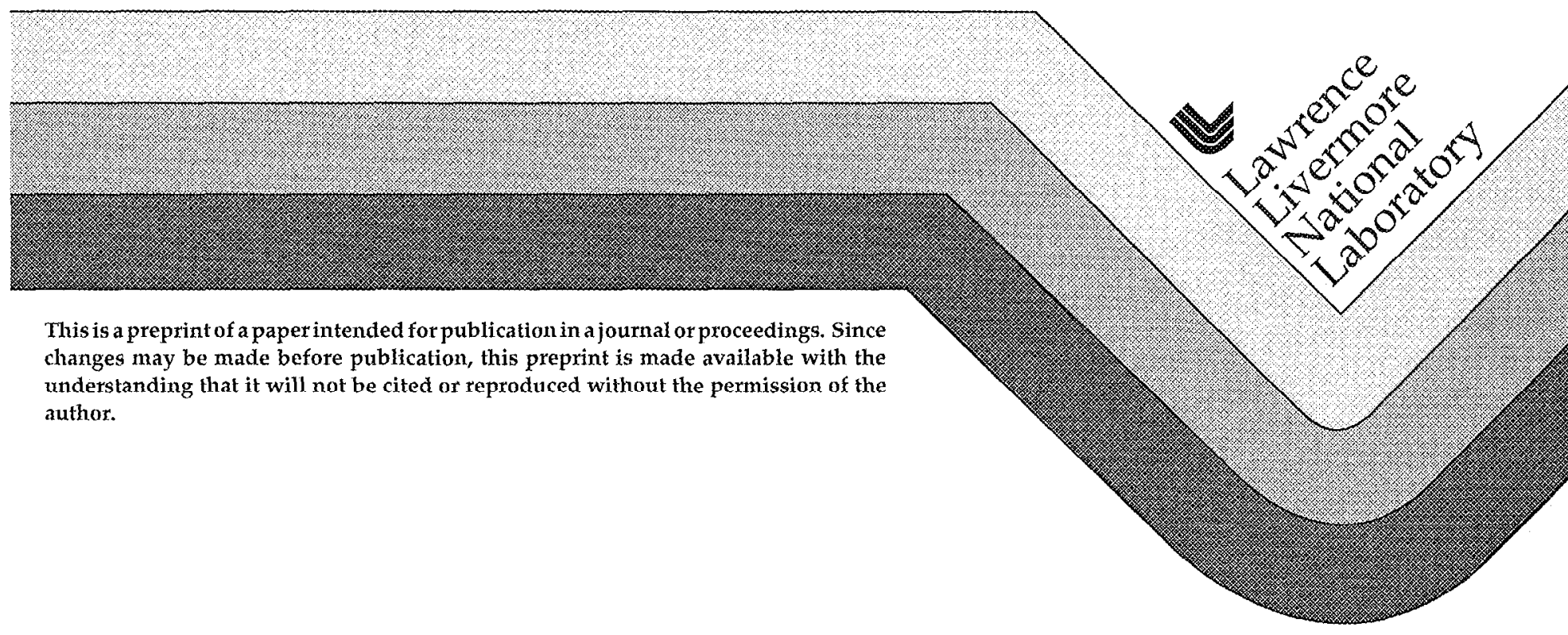




\section{DISCLAIMER}

This document was prepared as an account of work sponsored by an agency of the United States Government. Neither the United States Government nor the University of California nor any of their employees, makes any warranty, express or implied, or assumes any legal liability or responsibility for the accuracy, completeness, or usefulness of any information, apparatus, product, or process disclosed, or represents that its use would not infringe privately owned rights. Reference herein to any specific commercial product, process, or service by trade name, trademark, manufacturer, or otherwise, does not necessarily constitute or imply its endorsement, recommendation, or favoring by the United States Government or the University of California. The views and opinions of authors expressed herein do not necessarily state or reflect those of the United States Government or the University of California, and shall not be used for advertising or product endorsement purposes. 


\title{
TURBULENCE STUDIES IN TOKAMAK BOUNDARY PLASMAS WITH REALISTIC DIVERTOR GEOMFTRY*
}

\author{
X.Q. XU, R.H. COHEN, G.D. PORTER, T.D. ROGNLIEN, \\ D.D. RYUTOV, J. R. MYRA ${ }^{1}$, D. A. D'PPPOLITO' ${ }^{1}$, R. MOYER \\ Lawrence Livermore National Laboratory, \\ Livermore, California, \\ United States of America,
}

Abstract

Results are presented from the 3D nonlocal electromagnetic turbulence code BOUT [1] and the linearized shooting code BAL[2] to study turbulence in tokamak boundary plasmas and its relationship to the $L-H$ tansition, in a realistic divertor plasma geometry. The key results include: (1) the identification of the dominant resistive $X$-point mode in divertor geometry and (2) turbulence suppression in the $\mathrm{L}-\mathrm{H}$ transition by shear in the $\mathbf{E} \times \mathbf{B}$ drift speed, ion diamagnetism and finite polarization. Based on the simulation results, a parameterization of the transport is given that includes the dependence on the relevant physical parameters.

\section{Boundary Plasma Turbulence Studies}

BOUT models the boundary plasma that spans the separabrix using fluid equations for plasma vorticity, density, ion temperature and parallel momentum, and electron temperature and parallel momentum. The basic set of equations is described in Ref. [3] with extension to include magnetic pumping term by replacing the scalar pressure with the anisotropic pressure $\overleftrightarrow{\mathbf{P}}_{i}=P_{i} \overleftrightarrow{\mathbf{I}} \ldots$ $3 \eta_{0}\left(\left(V_{E \times B}+\left(V_{\nabla P_{i}}\right) \cdot \nabla \ln B\right)(\mathbf{b b} \quad \mathbf{I})\right.$, where $\eta_{0}$ is the classical parallel viscos ity. This reduced equation set includes the well-known linear instability drives for (1) Conducting-wall modes, (2) Kolvin-Helmholtz modes, (3) Curvature driven ideal MHD ballooning and resistive ballooning modes, (4) resistive drift $\Lambda$ lfven modes, (5) $\nabla_{x} v_{\|}$modes, and, $(6)$ axial shear modes $\left(\nabla_{\| \mathbf{E}} \times \mathbf{B}\right)$. In order to investigate pedestal physics, we first run the edge plasma transport code UEDGE/EFIT to get the X-point magnetic geometry and plasma profiles.

The BAL code is a linear eigenvalue code that solves a similar set of fluid equations as BOUT on field lines in the edge and SOL plasma for realistic $\mathrm{X}$-point geometry. The code implements the eikonal approximation, retaining drift, resistive and collisionless skin effects. We employ BAL to gain insight into the physics of the underlying linear instabilities in boundary plasmas, to benchmark the $3 \mathrm{D}$ turbulence code BOUT, and to search for various instability thresholds.

In an X-point divertor geometry, the local pitch $q(r, \theta)$ varies along the magnetic field line and there exists a steep parallel gradient of $q$ near the $X$ point. There are two effects: (1) local magnetic shear $\hat{s}(r, \theta)$ becomes large near the X-point; (2) the magnetic connection length $\left(L_{\|} \sim q(r, \theta) R\right)$ becomes infinite when a flux tube passes near an X-point. The first effect yields rapid mode oscillation along the field line near the X-point, and the validity of the eikonal representation becomes questionable. However, the parallel electron viscous damping and heat conduction terms smooth rapid $k_{\|}$variations near the $\mathrm{X}$-points. In the electromagnetic regime $\left(\epsilon=k_{\perp}^{2} c^{2} / \omega_{p e}^{2}<1\right)$, we find that normally the dissipation terms are smaller than the others by $\mathcal{O}(\epsilon)$. However,

* Work performed for USDOE under contracts W-7405-ENG-48 at LLNL, DE-FG0397ER54392 at LRC, DE-FG03-95ER54294 at UCSD. ${ }^{1}$ Lodestar Research Corporation, Boulder, CO 80301 USA; $^{2}$ University of California, San Diego, La Jolla, CA 92093 USA 
near the X-point(s), $\epsilon$ may exceed one because $k_{r}$ is large, dissipation can become important, and the modes become electrostatic. The localization of dissipation and electron skin effects near the $X$-points enables resistive curvature driven modes with strong drive near the X-point(s). This physics profoundly affects the unstable spectrum, and generally results in modes being localized between the upper and lower X-points on the low-ficld-side. Thus we refer to it as the "resistive X-point modes". The eigenmode structure from a typical BOUT simulation is shown in Fig. 1. The fluctuating potential is peaked near the low X-point just inside the separatrix. As shown in Fig. 1, even though the geometry is a single null divertor, there is still an X-point near the scrape offlayer (SOL) on the top of the machine, and its magnetic shear limits penetration of the fluctuations into inside of the torus. BOUT runs with the curvature term turned off show the significant reduction of linear instability growth rate. Linear eikonal ballooning analysis BAI, also shows that the X-point dramatically affects the eigenfunction shape and unstable spectrum. Fig. 2 shows eigenfunction and linear growth rate in SOL with and without X-points (in the latter case, $B_{p}$ and magnetic shear $\hat{s}$ are set to the mid-plane values). The fluctuation peaks near X-points and linear growth rate is typically smaller with the X-point. present. In the case without $X$-point, the eigenfunction peaked near where the lower X-point would have been since the curvature drive is strong there; the conduction-wall-mode is unstable, but is subdominanted where the magnetic field line normally intercepts the conducting plates. The frequency spectra of the mid-plane particle flux from probe measurements and BOUT simulations in L. mode shows reasonable agreement [3].

\section{L-H Transition}

For given DIII-D discharge 89840, we analyze the instabilities and turbulence at two different time slices $2370 \mathrm{~ms}$ and $2470 \mathrm{~ms}$, corresponding to $\mathrm{L}$ and H mode. We obtain equilibrium plasma profiles by using hyperbolic tangent fits to the mid-plane experimental data for the plasma density $N_{i 0}$, electron temperature $T_{e 0}$, ion temperature $T_{i 0}$ and assuming uniform profiles along the field line. We find that there is little change in the magnetic geometry in $\mathrm{L}$ and H mode. The plasma density $N_{i 0}$ is radially steepened by a factor of 4 , the ion temperature is steepened by a factor of 2 , the electron temperature is steepened by a factor of 1.5 from $\mathrm{l}$, to $H$ mode.

From BAL calculations, we find that in the DIIL-D $\mathrm{H}$ phase, the ideal MHD edge ballooning mode is near the marginal stability boundary and is a good candidate for the quasi-coherent mode seen in the experiments [4]. In both the $L$ and $H$ phases, a broad range of unstable toroidal mode numbers $n$ was found for resistive $\mathrm{X}$-point modes, in the range $n \sim 30-400$. The most linearly unstable growth rate and mode number $n$ are benchmarked between two codes BOUT and BAL in the L-mode phase. After averaging the linear growth rate on different flux surfaces from BAL over a half radial mode width from BOUT, the two codes agree very well.

In order to investigate the physics influences on the electric field $E_{r}$, we start from the Braginskii force balance equation:

$$
\mathbf{E}_{\perp}=\frac{1}{\mathbf{Z}_{\alpha} \mathbf{e} \mathbf{n}_{\alpha}} \nabla \cdot \mathbf{P}_{\alpha}-\frac{1}{\mathbf{c}} \mathbf{V}_{\alpha} \times \mathbf{B}-\frac{\mathbf{m}_{\alpha}}{\mathbf{Z}_{\alpha} \mathbf{e}}\left(\frac{\partial \mathbf{V}_{\alpha}}{\partial \mathbf{t}}+\mathbf{V}_{\alpha} \cdot \nabla \mathbf{V}_{\alpha}\right)
$$

This equation can be separated into equilibrium and turbulent parts $\mathbf{E}_{\perp}=$ $\mathbf{E}_{\perp 0}+\delta \mathbf{E}_{\perp}$ :

$$
\begin{gathered}
\mathbf{E}_{\perp 0}-\frac{1}{\mathbf{Z}_{\alpha} \mathbf{e} \mathbf{n}_{\alpha}} \nabla \cdot \mathbf{P}_{\alpha 0}-\frac{1}{\mathbf{c}} \mathbf{V}_{\alpha}^{0} \times \mathbf{B}_{\mathbf{0}} \\
\delta \mathbf{E}_{\perp}=\frac{\mathbf{1}}{\mathbf{Z}_{\alpha} \mathbf{e} \mathbf{n}_{\alpha}} \nabla \cdot \delta \mathbf{P}_{\alpha}-\frac{\mathbf{1}}{\mathbf{c}} \delta\left(\mathbf{V}_{\alpha} \times \mathbf{B}\right)-\frac{\mathbf{m}_{\alpha}}{\mathbf{Z}_{\alpha} \mathbf{e}}\left(\frac{\partial \delta \mathbf{V}_{\alpha}}{\partial \mathbf{t}}+\delta\left(\mathbf{V}_{\alpha} \cdot \nabla \mathbf{V}_{\alpha}\right)\right)
\end{gathered}
$$


The equilibrium electric field $\mathbf{E}_{\perp 0}$ is calculated either from measured plasma profiles or from the edge plasma transport code UEDGE [5] (which solves the current continuity equation $\nabla \cdot n_{i} e\left(\mathbf{V}_{i}^{0}-V_{e}^{0}\right)=0$ and $V_{\perp}^{0}$ are iteratively determined from Eq. (2)). Instead of calculating $\delta \mathbf{E}_{\perp}$ from $\mathrm{Eq}$. (3), the turbulent $\delta \phi$ is obtained from quasineutrality $\left(\delta n_{e}=\delta n_{i}\right)$; submiting Eq. (3) for the electron and ion species into the current continuity equation $(\nabla \cdot \delta \mathfrak{I}=\mathbf{0})$ gives the vorticity equation. The calculated electric field $E_{r}$ on mid-plane from BOUT is given in Fig. 3 for the H-mode phase. It is shown that the overall radial profile of $E_{r}$ is determined by equilibrium force balance equation; however, the turbulence produces significant polnidal flow just inside the separatrix. The $E_{r}$ in $\mathrm{L}$-mode is small and positive. The turbulent suppression of fluctuating potential $\langle\delta \phi\rangle_{\text {rms }}$ and ion heat diffusivity $\chi_{i}$ are shown in Fig. 4 and 5 . Fven in the absence of flow shear, the SOL transport is moderated due to finite ion diamagnetism and finite polarization stabilization of SOL modes [6]. Flow shear reduces turbulence both in the SOL and edge regions $[7][8]$.

\section{L-mode Transport Scalings}

The turbulent transport coefficients can be expressed in the form: $\chi_{\perp}=$ $\chi_{G B} F\left(\hat{\beta}, \rho_{s} / R_{0}, L_{1} / \rho_{s}, L_{\|} / \rho_{s}, \hat{s}, \hat{\nu}, \Lambda\right)$. Here $\chi_{G B}=\left(c T_{e} / e B_{0}\right)\left(\rho_{s} / L_{\perp}\right)$ is the gyro-Bohm coefficient, and $L_{\perp}$ represents the set of scale lengths of density, temperature, parallel velocity and ion pressure gradient perpendicular to $\mathbf{B}$. $F$ is a dimensionless function of the dimensionless arguments determined from theoretical arguments and/or from simulations. The dependence of $\chi_{i}$ on $\hat{\beta}$ is plotted in Fig. 7, the dependence of $\chi_{i}$ on $\nu_{e i}$ is given in Fig. 8, and the dependence of $\chi_{i}$ on $L_{n}$ is given in Fig. 9 .

\section{Summary}

Our 3D nonlocal fluid turbulence simulations have been extended for boundary plasmas into the experimentally relevant $X$-point divertor geometry. A broad range of high toroidal mode number $n$ for resistive $X$-point turbulence is found in the range $n \sim 30-400$ both for L-mode and H-mode. The localization of electron skin effects near the X-points enables resistive curvature driven modes with strong drive near the $\mathrm{X}$-point(s). Flow shear is found to be the dominant mechanism for the suppression of boundary turbulence in edge (as well as SOL). Turbulence simulations yield a visible poloidal flow just inside the magnetic separatrix. The scaling scans of the transport show the sensitivities of $\chi_{i}$ on $\hat{\beta}, \nu_{e i}$ and $L_{n}$.

\section{REFERENCES}

[1] Xu, X. Q., and Cohen, R. H., Contributions to Plasma Physics, June(1998).

[2] Myra, J. R., D'Tppolito, D. A., and Goedbloed, J. P., Physics of Plasmas, Vol.4 1330-1341(1997).

[3] Xu, X. Q., Cohen, R. H., PORTER,G. D., Myra, J. R., D'Ippolito, D. A., Moyer, R., 13 th Iternational Conference on Plasma Surface Interactions in Controlled Tusion Devives, San Diego, California, May (1998).

[4] T. Rhodes and C. Rettig, private communication; Moyer, R., et al., APS Division of Plasma Physics, Pittsburgh, PA, Poster, hTupP2.32(1997).

[5] T.D. Rognlien, G.D. Porter, and D.D. Ryutov, 13th Iternational Conference on Plasma Surface Interactions in Controlled Fusion Devives, San Diego, California, May(1.998).

[6] Cohen, R. H., and Xu, X. Q., Physics of Plasmas Vol.2 3374-3383(1995).

[7] Biglari, H., Diamond, P. H., and Terry, P. W., Phys. Fluids P 2 (1990) 1.

[8] Burrell, K. H.,Physics of Plasmas Vol.4 (1997) 1.499. 


\section{FIGURE CAPTIONS}

Fig. 1. Fluctuating potential $e\langle\phi\rangle_{\varphi} / T_{e 0}$ in $2 \mathrm{D}$ radial and poloidal plan from a typical BOUT simulation.

Fig. 2. (a) Fluctualing potential with and without X-point; (b) linear growth rate with and without $\mathrm{X}$-point.

Fig. 3. Electric field $E_{r}(x)$ in $\mathrm{H}$ mode from BOUT simulations. The solid line is the compuled $E_{r}(x)$ including the turbulence-generated contribution, the dashed line is the equilibrium electric field calculated from the smoothed diamagnetic flow and the sheath potential in the SOL, The dot from CER measurements.

Fig. 4. Electrostatic potential fluctuations $e\langle\phi\rangle_{\varphi} / T_{e 0}$ across the separatrix measured at mid-plane from BOUT simulalioms

Fig. 5. Turbulence-generated ion heal diffusivity $\chi_{i}$ from BOUT simulations.

Fig. 6. Scaling with $\hat{\beta}=\beta / 2 \mu_{e}, \mu_{e}=m_{e} / M_{i}, \beta=8 \pi n T_{e} / B^{2}$, of the ion heat diffusivity $\chi_{i}$ from the $\mathbf{E} \times \mathrm{B}$ contribution. 


\section{Fluctuating Potential Indicates that Mode is electrostatic near X-points}

(Lnear mase)

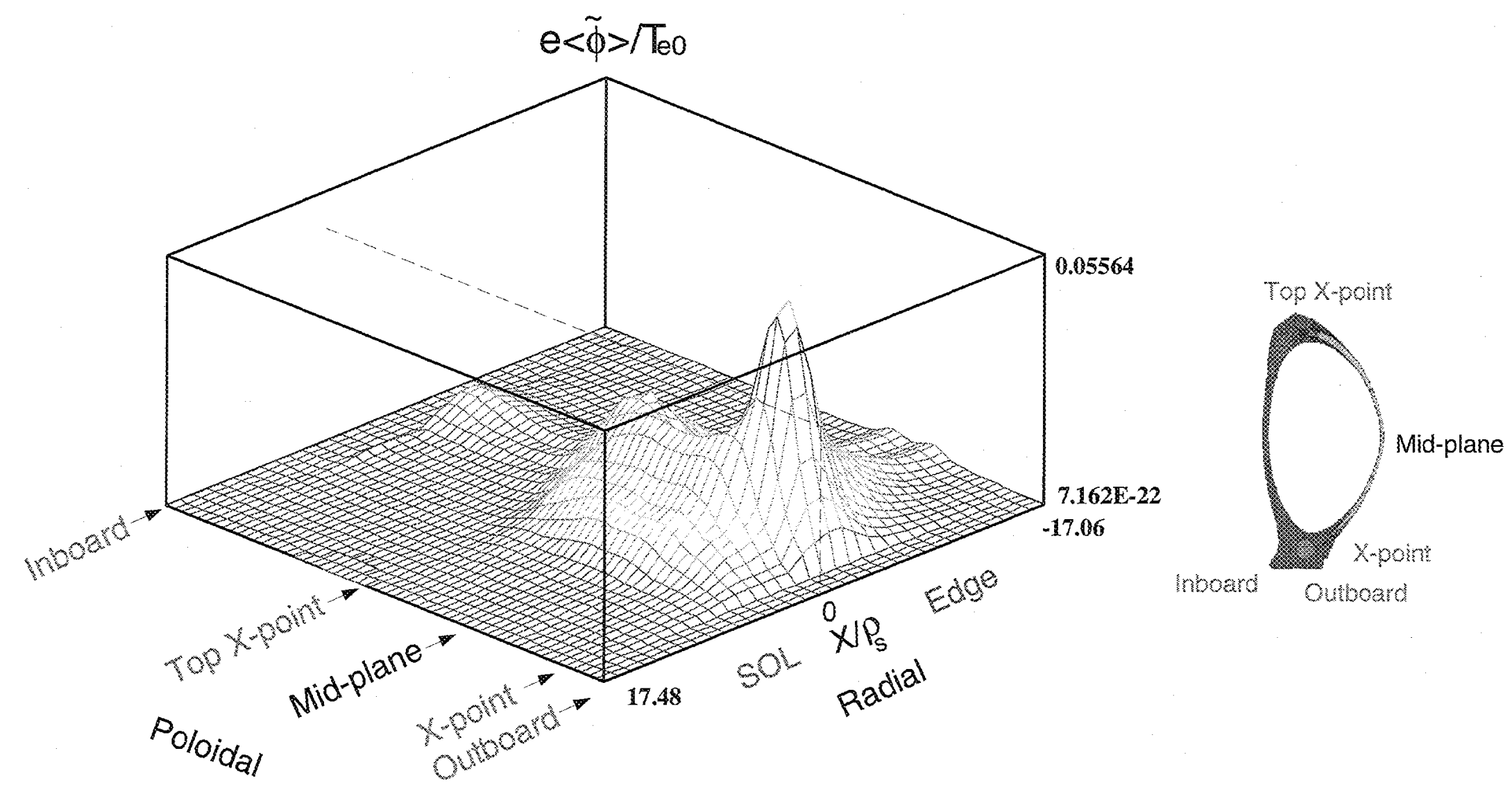




\section{The X-point dramatically affects the eigenfunction shape and unstable spectrum:}

- L-mode SOL case shown

- "no X-pt" case artificially sets Bp and magnetic shear to midplane values

- X-point prevents mode from reaching divertor plate

- net effect of X-point is stabilizing (more line bending and less weighting of bad curvature)

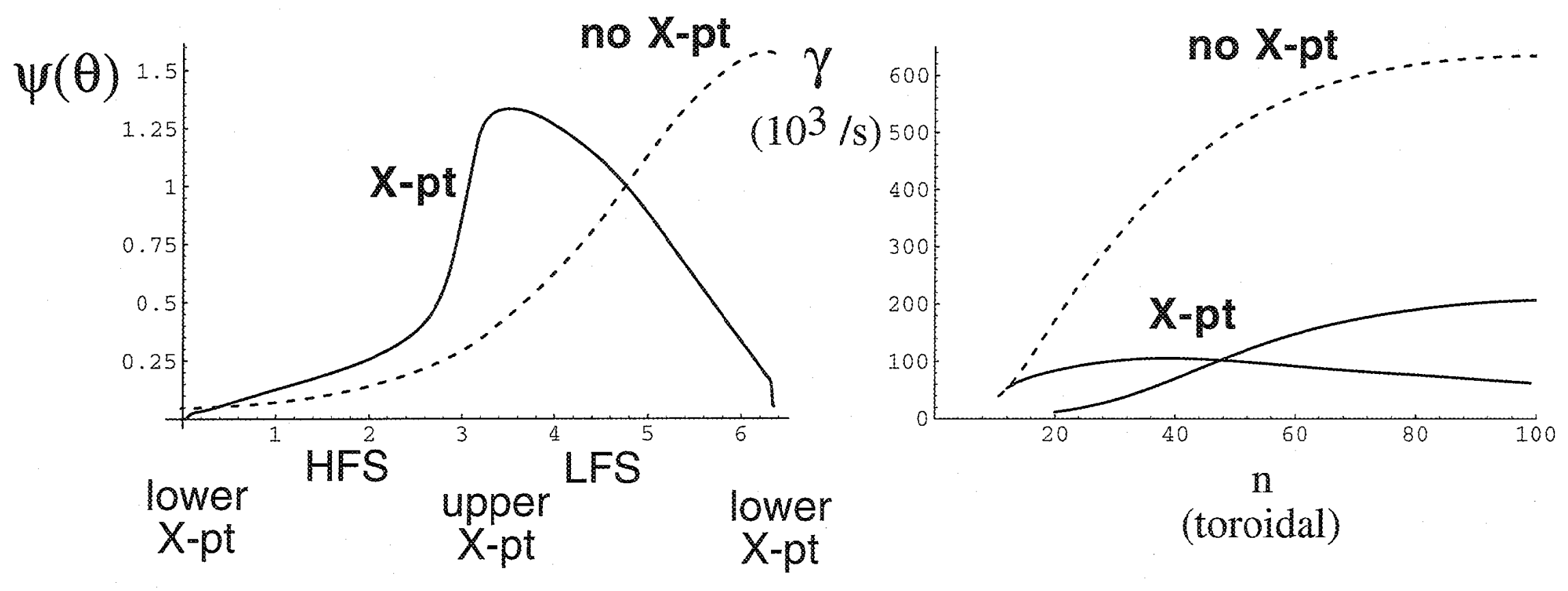




\section{Elecric Fidd Profiles across Magnetic Sepatru at Midmplane in DII-D in Momode}

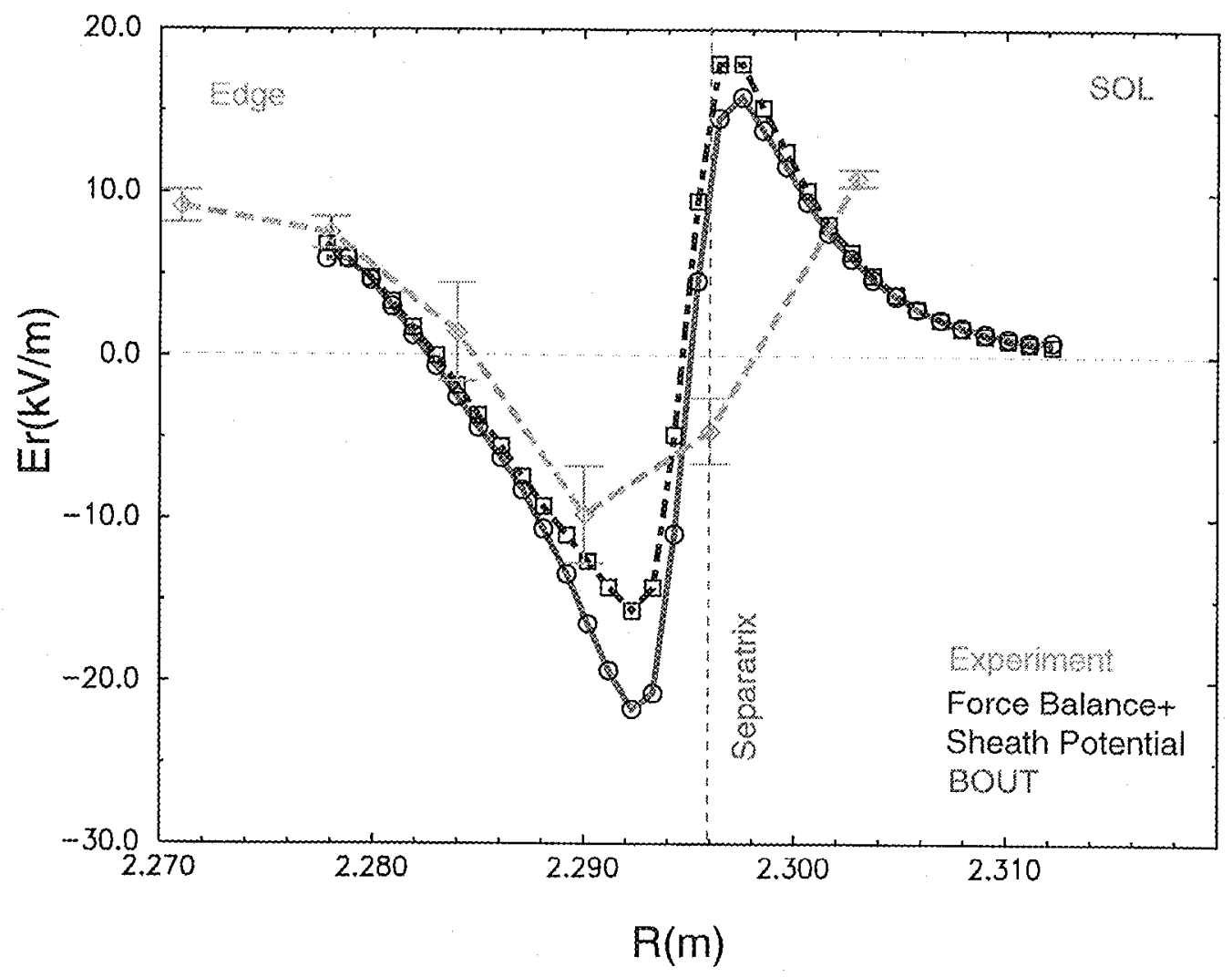




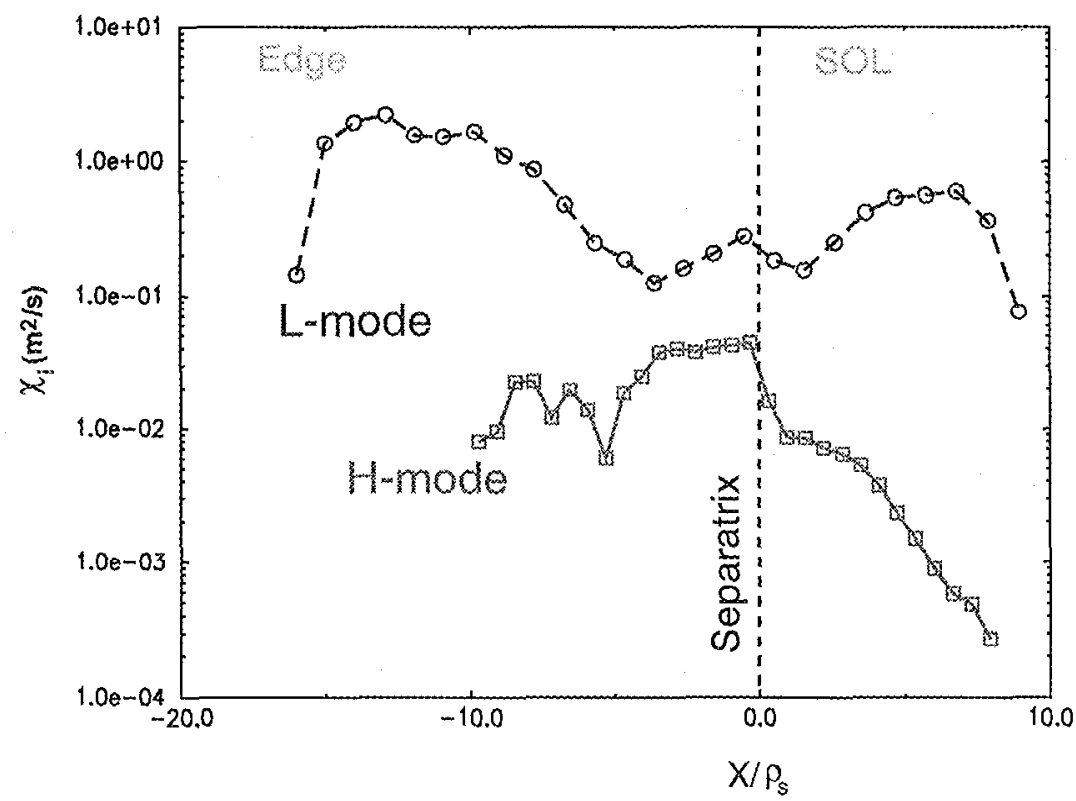

Ix lon heat difusivity reduced in H-mode due to poloidal flow shear, ion diamagnetism + finite polarization 
Electrostatic Potential Fluctuations across the Separatrix Measured at Mid-plane from BOUT Simulations for DIIl-D discharge 89840

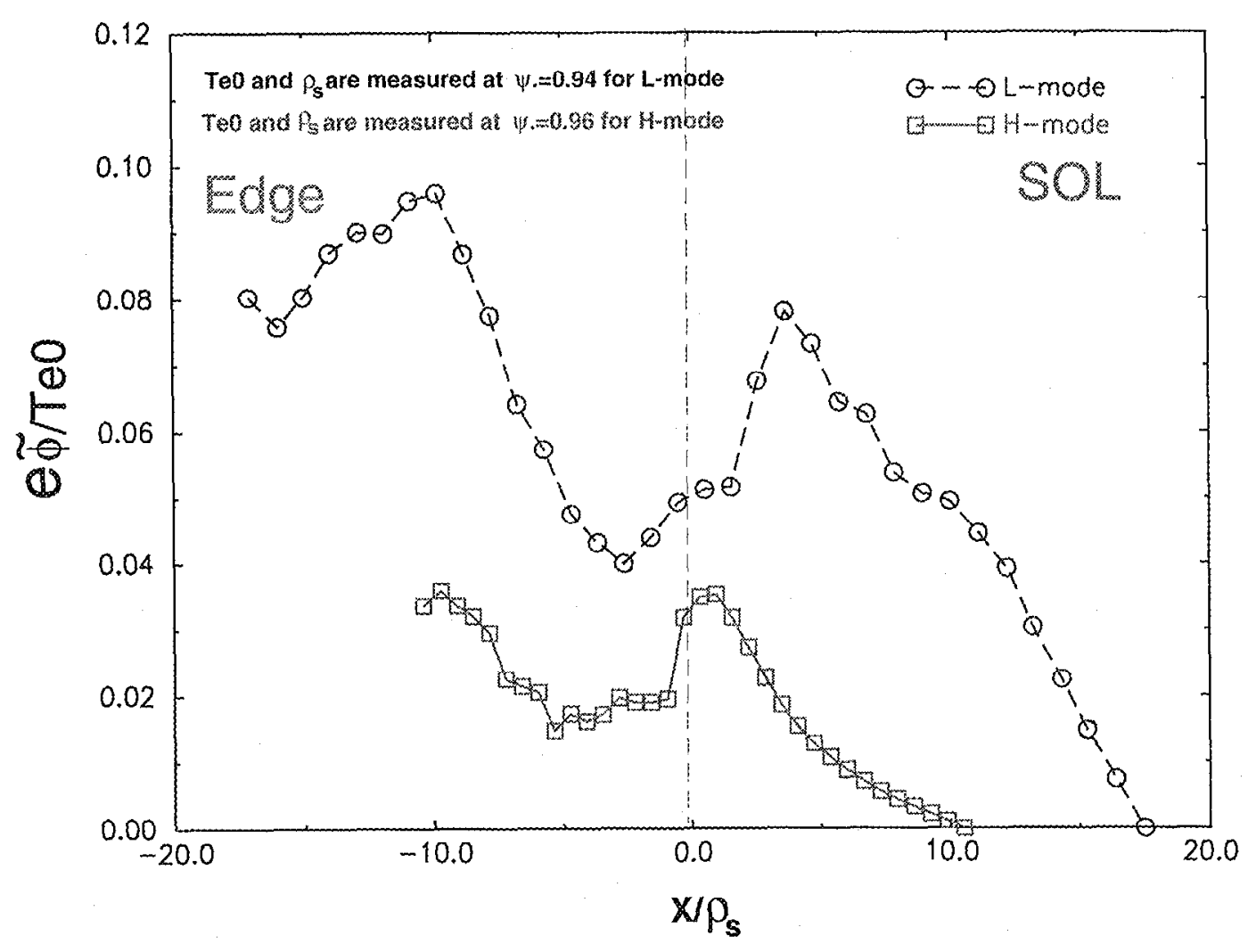




\section{Scalings with $\hat{\beta}$ of the Diffusivities are Favourable}

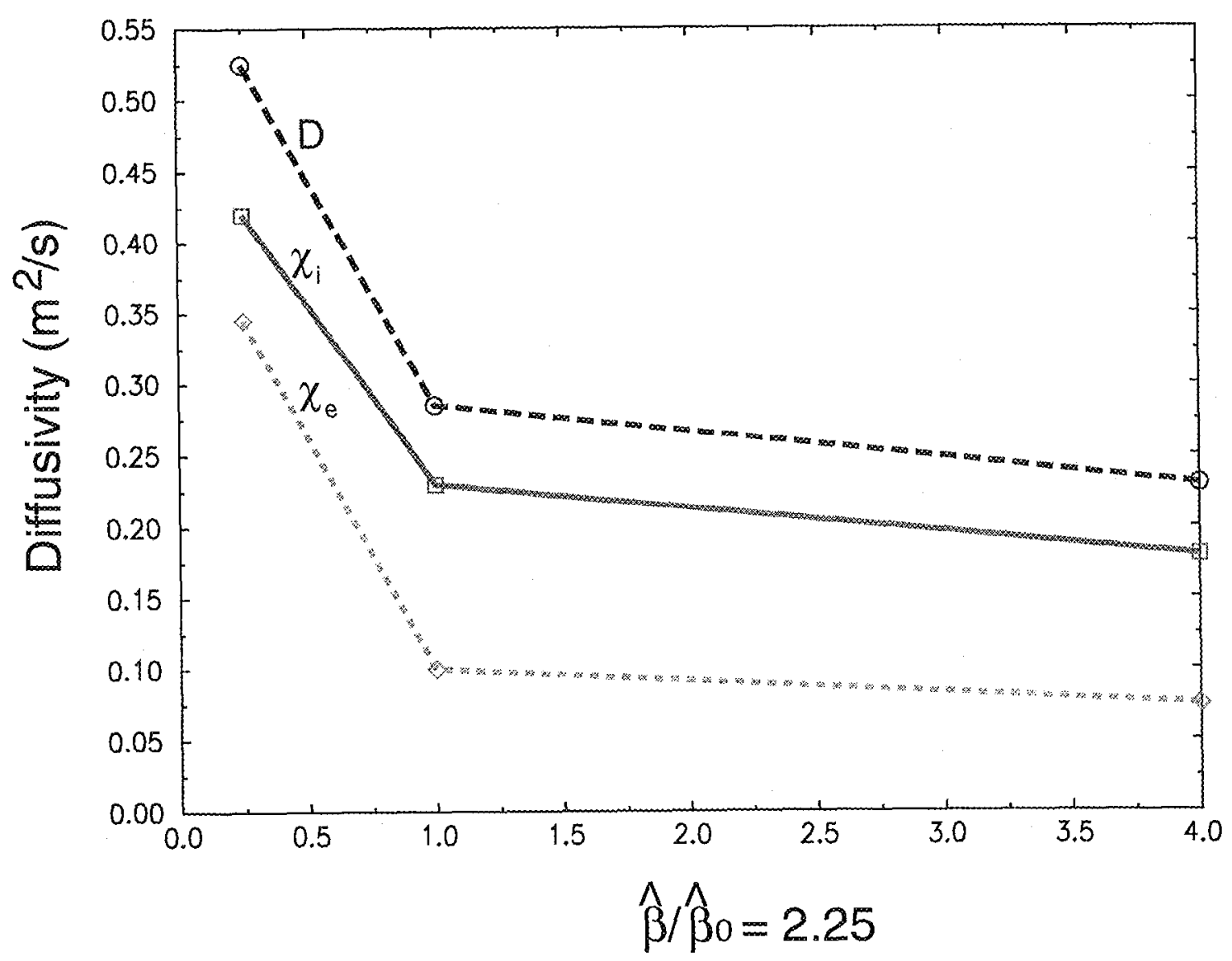

